\title{
Barreiras percebidas para a prática de atividade física em universitários da área da saúde de uma instituição de ensino superior da cidade de Fortaleza, Brasil
}

\author{
Perceived barriers to the practice of physical activity in University \\ Students of Health Sciences of a higher education institution in the city of \\ Fortaleza, Brazil
}

Tiago Pereira do Nascimento', Felipe Rocha Alves², Evanice Avelino de Souza²

\begin{abstract}
Resumo
O objetivo deste estudo foi analisar a prática de atividade física e as principais barreiras percebidas, bem com investigar sua associação com variáveis sociodemográficas, em universitários da área da saúde. Estudo transversal realizado com 736 universitários ( $\leq 18$ e $\geq 36$ anos de idade) de ambos os sexos, de uma instituição de ensino superior da cidade de Fortaleza, Brasil. Foram coletadas informações sociodemográficas, prática de atividade ( $\operatorname{sim}$ ou não) e barreiras percebidas. O teste do Qui-quadrado foi utilizado para associação das variáveis sociodemográficas com as principais barreiras percebidas e a prática de atividade física, adotando-se $\mathrm{p} \leq 0,05$. As principais barreiras percebidas foram: jornada de estudos extensa $(33,4 \%)$, falta de energia/cansaço $(20,7 \%)$, falta de companhia $(18,1 \%)$, jornada de trabalho extensa $(17,8 \%)$ e falta de interesse $(17,0 \%)$. Essas barreiras foram associadas ao semestre letivo $(\mathrm{p} \leq 0,001)$. A jornada de estudos extensa e jornada de trabalho extensa estiveram associadas ao sexo $(\mathrm{p} \leq 0,004)$ e a faixa etária $(p \leq 0,001)$. A prática de atividade física esteve associada ao sexo, curso e semestre letivo $(\mathrm{p} \leq 0,001)$. Conclui-se que a jornada de estudos extensa foi apontada como a principal barreira para a prática de atividade física entre os universitários. Em geral as principais barreiras percebidas eram de cunho social, comportamental e físicas. As principais barreiras percebidas estiveram associadas ao semestre letivo.
\end{abstract}

\section{Palavras-chave}

Atividade motora; Estudantes; Estilo de vida; Estudos transversais.

\section{Abstract}

This study aimed to analyze the physical activity and the main barriers perceived by university students, as well as to investigate Its association with sociodemographic variable. This was a Cross-sectional study with 736 university students ( $\leq 18$ and $\geq 36$ years old) of both genders, at a higher education institution in the city of Fortaleza, Brazil. Sociodemographic information, activity practice (yes or no) and perceived barriers were collected. The chi-square test was used to associate the sociodemographic variables with the main perceived barriers and the practice of physical activity, adopting $p \leq 0.05$. The main perceived barriers were extensive study bours (33.4\%), lack of energy / fatigue $(20.7 \%)$, lack of company (18.1\%), extensive workload (17.8\%) and lack of Of interest (17.0\%). These barriers were associated to the semester $(p \leq 0.001)$. The extensive study journey and extended working day were associated with gender and age group $(p \leq 0.004$ and $p \leq 0.001$ respectively). The practice of physical activity was associated to sex, course and semester $(p \leq 0.001)$. It was concluded that the extensive study periods was considered the main barrier to physical activity among university students. It is expected that these results will serve as a reference for the systematization of possible interventions in order to develop healthy living habits in university students. In geneval, the main perceived barriers were social, behavioral and physical. The main perceived barriers were associated with the semester.

\section{Keywords}

Motor Activity; barriers, students; Life style; Cross-sectional studies.

\section{Introdução}

Com a expansão do ensino superior, os universitá-

1 Faculdade Nordeste - FANOR. Departamento de Educação Física. Fortaleza, Ceará, Brasil.

2 Universidade Federal do Ceará - UFC. Faculdade de Medicina programa de pósgraduação em Ciências Médicas. Fortaleza, Ceará, Brasil. rios, a cada dia, vêm representando uma esfera da população brasileira. De acordo com o Censo do Ensino Superior realizado pelo Instituto Nacional de Estudos e Pesquisas Educacionais Anísio Teixeira, em dez anos, as matriculas em cursos superiores mais que 
dobraram: de 3.036.113, em 2001, passaram para 6.379.299, em $2010^{1}$.

Em função disso, das obrigações acadêmicas e profissionais, os universitários tendem a modificar seus hábitos de vida, assumindo algumas condutas nocivas à saúde, dentre elas, a diminuição do nível de atividade física $(\mathrm{AF})^{2}$. Estudo epidemiológico realizado na Europa com 19.298 universitários de 23 países relatou-se que a prevalência de inatividade física no tempo de lazer era de $23 \%$ (Noroeste da Europa e Estados Unidos), 30\% (Europa Central e Oriental), 39\% (Mediterrâneo), $42 \%$ (Ásia) e 44\% (Países em desenvolvimento) ${ }^{3}$. No Brasil, um estudo realizado na região sul, com amostra representativa de universitários de uma instituição pública, identificou nível insuficiente de prática de AF em 30,8\% dos estudantes ${ }^{4}$.

Adotar e manter um estilo de vida fisicamente ativo envolve múltiplas variáveis, tais como demográficas, biológicas, psicológicas, cognitivas, emocionais, culturais e sociais ${ }^{5,6}$. Alguns desses fatores representam barreiras para prática de AF, dificultando ou impendido a prática de $\mathrm{AF}^{7,8}$.

As barreiras percebidas podem ser de cunho sociocultural (conservadorismo, influência negativa do meio), pessoal (medo, preguiça, falta de tempo, apatia) e ambiental (falta de locais, equipamento e condições adequadas para a prática) ${ }^{9}$. Pesquisas com diferentes populações têm sido desenvolvidas no intuito de identificar as barreiras que dificultam a prática de AF regular ${ }^{8,11}$. Poucos estudos abordaram essa temática em estudantes universitários brasileiros ${ }^{12,13}$, reforçando a necessidade de intensificação de pesquisas que objetivem identificar possíveis barreiras percebidas para prática de $\mathrm{AF}$ em universitários. Acredita-se que os resultados destes estudos poderão ajudar a orientar profissionais da saúde e educação envolvidos com o público universitário no direcionamento de ações e programas de promoção de AF. Com base nesse panorama, o presente estudo teve como objetivo analisar a prática de atividade física e as principais barreiras percebidas, bem com investigar sua associação com variáveis sociodemográficas, em universitários da área da saúde.

\section{Métodos}

O estudo apresentou delineamento transversal e foi realizado entre outubro e novembro de 2015. O protocolo do estudo foi aprovado pelo Comitê de Ética em Pesquisa da Faculdade Metropolitana da Grande Fortaleza, Ceará (Processo n ${ }^{\circ} 1$. 416. 943).

A pesquisa envolveu universitários da área da saúde regularmente matriculados em uma instituição de ensino superior da cidade de Fortaleza, Ceará. Trata-se do único centro universitário com padrão internacional do estado do Ceará, fazendo parte de um grupo de ensino norte-americano, presente em 40 países, com mais de 110 mil alunos, além de possuir um expressivo corpo discente nos cursos da área da saúde.

Segundo a secretaria da instituição no ano de 2015 (segundo semestre) existiam 1.500 universitários regularmente matriculados na área da saúde, distribuídos nos seguintes cursos: educação física $(n=126)$; enfermagem $(n=427)$; fisioterapia $(\mathrm{n}=296)$ e nutrição $(\mathrm{n}=651)$.

Primeiramente, foi entregue as coordenações dos cursos da área da saúde o termo de anuência para autorização da pesquisa. Não havendo oposição dos coordenadores em relação à realização do estudo na instituição, foi agendada uma data para a apresentação da pesquisa aos universitários (exposição dos objetivos e relevância do estudo, e esclarecimento de possíveis dúvidas). Em uma terceira visita a instituição, deu-se início a aplicação dos questionários com os universitários que estavam presentes em sala de aula nos dias de coleta. 
Para a realização do presente estudo foram convidados todos os universitários da área da saúde, devido as coordenações dos cursos informarem que muitos deles, principalmente nos cursos de enfermagem e fisioterapia já haviam iniciado as disciplinas de estágio e ficariam ausentes na instituição. Por isso, na coleta adotou-se o seguinte procedimento: duas semanas, para aplicação nas turmas de primeiro e segundo semestre, outras duas semanas para as turmas de terceiro, quarto e quinto semestre e na quinta e sexta semana, os semestres finais.

A coleta de dados foi realizada por estudantes do curso de Educação Física, pertencentes ao Grupo de Pesquisa em Saúde e Atividade Física na Escola (UFC), após treinamento teórico-prático e realização de coleta supervisionada no estudo piloto.

As variáveis sociodemográficas sexo, faixa etária, curso, semestre letivo (Semestres iniciais: $1^{\circ}$ e $2^{\circ}$ semestre; Semestres intermediários: $3^{\circ}$ ao $6^{\circ}$; Semestres finais: $7^{\circ}$ em diante) e trabalho (trabalham e/ou estejam estagiando com vínculo empregatício) foram autorrelatadas. A informação referente a prática de AF foi obtida mediante a seguinte pergunta: você pratica atividade física regular (sim ou não)?

As barreiras foram mensuradas através do questionário proposto por Martins e Petroski ${ }^{14}$, no qual foi acrescentado o item "jornada de estudos extensa”, para melhor validade junto aos discentes. O questionário foi composto por vinte barreiras (jornada de estudos extensa, jornada de trabalho extensa, compromissos familiares, falta de clima adequado, falta de espaço disponível para a prática, falta de equipamentos disponíveis, tarefas domésticas, falta de companhia, falta de incentivo da família e/ou amigos, falta de recursos financeiros, mau humor, medo de lesionar-se, limitações físicas, dores leves ou mal-estar, falta de energia, falta de habilidades físicas, falta de conhecimento ou orientação sobre atividade física, ambiente insuficientemente seguro, preocupação com a aparência durante a prática, falta de interesse em praticar), acrescentando-se o item outras. Considerando as barreiras listadas, os universitários deveriam indicar a frequência que acontecia à barreira (barreira sempre presente $=5$, barreira quase sempre presente $=4$, barreira às vezes presente $=3$, barreiras raramente presente -2 e para barreira nunca presente $=1$ ). Foi considerado como uma "barreira percebida", quando os universitários referiram "sempre" ou "quase sempre" um empecilho para a prática de AF.

Para análise dos dados foi utilizada a distribuição por frequências absoluta (n) e relativa (\%) para todas as variáveis analisadas. O teste do Qui-quadrado foi utilizado para a análise da associação das variáveis (sexo, curso, faixa etária, semestre letivo e ocupação profissional) com as principais barreiras percebidas e a prática de AF, sendo que para variável curso adotou-se a correção de Yates por algumas frequências de respostas terem sido menores do que cinco. O nível de significância foi estabelecido em $<\mathbf{5 \%}(\mathrm{p} \leq 0,05)$. Todos as análises foram realizadas no software IBM SPSS ${ }^{\mathrm{R}}$ Statistics 21.0 .

\section{Resultados}

Todos os 1500 universitários matriculados, foram convidados a participar da pesquisa. Contudo, 91 não responderem o questionário corretamente, 35 se recusaram a participar e 638 não estavam presente nos dias da coleta. Sendo assim, à mostra final deste estudo foi constituída por 736 universitários (169 homens e 567 mulheres). A maioria dos universitários tinha entre 19 e 25 anos $(73,8 \%)$, pertencia ao curso de nutrição $(43,3 \%)$, estava matriculada nos semestres intermediários (61,1\%), não trabalhava (71,1\%) e praticava $\mathrm{AF}(59,1 \%)$ - Tabela 1.

Em relação à prática de $\mathrm{AF}$, os resultados indicaram que os universitários do sexo masculino $(56,1 \%, \mathrm{p} \leq 0,001)$, os estudantes de educação física $(71,0 \%, \mathrm{p} \leq 0,001)$ 
e os matriculados em semestres iniciais $(61,5 \%, \mathrm{p} \leq 0,001)$ apresentaram uma maior participação com a prática regular de AF.

TABELA 1 - Características da amostra em relação ao sexo, faixa etária, curso, semestre letivo, trabaIho e prática de atividade física dos universitários, Fortaleza, 2015 ( $n=736)$.

\begin{tabular}{lll}
\hline Variáveis & $n$ & $\%$ \\
\hline Sexo & & \\
\hline Masculino & 169 & 23,0 \\
\hline Feminino & 567 & 77,0 \\
\hline Faixa etária & & \\
\hline$\leq 18$ & 79 & 10,7 \\
\hline 19 a 25 & 543 & 73,8 \\
\hline 26 a 35 & 100 & 13,6 \\
\hline$\geq 36$ & 14 & 1,9 \\
\hline Curso & & 8,5 \\
\hline Educação Física & 62 & 28,5 \\
\hline Enfermagem & 210 & 19,7 \\
\hline Fisioterapia & 145 & 43,3 \\
\hline Nutrição & 319 & \\
\hline Semestre letivo & & 24,8 \\
\hline Iniciais & 182 & 61,1 \\
\hline Intermediários & 450 & 14,1 \\
\hline Finais & 104 & 70,9 \\
\hline Trabalha & & 59,1 \\
\hline Sim & 213 & \\
\hline Não & 523 & \\
\hline Prática de atividade física & & \\
\hline Sim & 301 & \\
\hline Não & & \\
\hline & & \\
\hline
\end{tabular}

TABELA 2 - Comparação da proporção de praticantes de atividade física regular de acordo com sexo, faixa etária, curso, semestre letivo e trabalho, Fortaleza, $2015(n=736)$.

\begin{tabular}{|c|c|c|c|}
\hline \multirow{2}{*}{ Variáveis } & \multicolumn{2}{|c|}{ Praticantes de AF } & \multirow[t]{2}{*}{$p^{*}$} \\
\hline & $n$ & $\%$ & \\
\hline \multicolumn{4}{|l|}{ Sexo } \\
\hline Masculino & 97 & 56,1 & $\leq 0,001$ \\
\hline Feminino & 204 & 36,0 & \\
\hline \multicolumn{4}{|l|}{ Faixa etária } \\
\hline$\leq 18$ & 41 & 51,9 & \\
\hline 19 a 25 & 227 & 41,8 & 0,011 \\
\hline 26 a 35 & 28 & 28,0 & \\
\hline$\geq 36$ & 5 & 35,7 & \\
\hline \multicolumn{4}{|l|}{ Curso } \\
\hline Educação Física & 44 & 71,0 & \\
\hline Enfermagem & 48 & 22,9 & $\leq 0,001$ \\
\hline Fisioterapia & 58 & 40,0 & \\
\hline Nutrição & 151 & 47,3 & \\
\hline \multicolumn{4}{|l|}{ Semestre letivo } \\
\hline Iniciais & 112 & 61,5 & \\
\hline
\end{tabular}

Continua. . 
. continua

\begin{tabular}{lccc}
\hline \multirow{2}{*}{ Variáveis } & $\mathrm{n}$ & Praticantes de AF & $\mathrm{p}^{*}$ \\
\cline { 2 - 4 } & 166 & 36,9 & \\
\hline Intermediários & 23 & 22,1 & $\leq 0,001$ \\
\hline Finais & & & \\
\hline Trabalha & 79 & 37,1 & 0,180 \\
\hline Sim & 222 & 42,4 & \\
\hline Não & &
\end{tabular}

* Teste do Qui-quadrado

As principais barreiras percebidas para a prática de $\mathrm{AF}$ foram: jorna de estudos extensa $(33,4 \%)$, falta de energia $(20,7 \%)$, falta de companhia para a prática $(18,1 \%)$, jornada de trabalho extensa $(17,8 \%)$ e falta de interesse em praticar $(17,0 \%)$ - Tabela 3.

TABELA 3 - Barreiras percebidas para a prática de atividade física em universitários da área da saúde. Fortaleza, $2015(n=736)$.

\begin{tabular}{lcc}
\hline \multicolumn{1}{c}{ Barreiras } & $n$ & $\%$ \\
\hline Jornada de estudos extensa & 246 & 33,4 \\
\hline Jornada de trabalho extensa & 131 & 17,8 \\
\hline Compromissos familiares & 94 & 12,8 \\
\hline Falta de clima adequado & 27 & 3,7 \\
\hline Falta de espaço disponível para a prática & 84 & 11,4 \\
\hline Falta de equipamentos disponíveis & 84 & 11,4 \\
\hline Tarefas domésticas & 95 & 12,9 \\
\hline Falta de companhia & 133 & 18,1 \\
\hline Falta de incentivo da família e/ou amigos & 86 & 11,7 \\
\hline Falta de recursos financeiros & 80 & 10,9 \\
\hline Mau humor & 41 & 5,6 \\
\hline Medo de lesionar-se & 35 & 4,8 \\
\hline Limitações físicas & 29 & 3,9 \\
\hline Dores leves ou mal-estar & 29 & 3,9 \\
\hline Falta de energia & 152 & 20,7 \\
\hline Falta de habilidades físicas & 59 & 8,0 \\
\hline Falta de conhecimento ou orientação sobre AF & 41 & 5,6 \\
\hline Ambiente insuficientemente seguro & 67 & 9,1 \\
\hline Preocupação com a aparência durante a prática & 37 & 5,0 \\
\hline Falta de interesse em praticar & 125 & 17,0 \\
\hline Outros & 6 & 0,8 \\
\hline
\end{tabular}

$\mathrm{Na}$ tabela 4, são apresentadas as cinco principais barreiras percebidas de acordo com sexo, faixa etária, curso e semestre letivo. As barreiras jornada de estudos extensa e jornada de trabalho extensa estiveram associadas ao sexo $(p \leq 0,004)$ e faixa etária $(\mathrm{p} \leq 0,001)$. Todas as barreiras estiveram associadas ao semestre letivo $(\mathrm{p} \leq 0,001)$ e apenas a jornada de trabalho extensa não esteve associada ao curso ( $p>0,05)$. 
TABELA 4 - Principais barreiras percebidas para a prática de atividade física por universitários da área da saúde de acordo com sexo, faixa etária, curso e semestre letivo, Fortaleza, 2015.

\begin{tabular}{|c|c|c|c|c|c|c|c|c|c|c|}
\hline \multirow{2}{*}{ Variáveis } & \multicolumn{2}{|c|}{$J E E^{* *}$} & \multicolumn{2}{|c|}{ Falta de energia } & \multicolumn{2}{|c|}{ Falta de companhia } & \multicolumn{2}{|c|}{$\mathrm{JTE}^{* * *}$} & \multicolumn{2}{|c|}{ Falta de interesse } \\
\hline & $n$ & $\%$ & $n$ & $\%$ & $n$ & $\%$ & $n$ & $\%$ & $n$ & $\%$ \\
\hline \multicolumn{11}{|l|}{ Sexo } \\
\hline Masculino & 41 & 5,6 & 23 & 3,1 & 20 & 2,7 & 36 & 4,9 & 18 & 2,4 \\
\hline Feminino & 205 & 27,9 & 129 & 17,5 & 113 & 15,4 & 95 & 12,9 & 107 & 14,5 \\
\hline$p^{*}$ & \multicolumn{2}{|c|}{$\leq 0,004$} & \multicolumn{2}{|c|}{0,010} & \multicolumn{2}{|c|}{0,016} & \multicolumn{2}{|c|}{0,175} & \multicolumn{2}{|c|}{0,012} \\
\hline \multicolumn{11}{|l|}{ Faixa Etária } \\
\hline$\leq 18$ & 23 & 3,1 & 12 & 1,6 & 12 & 1,6 & 4 & 0,5 & 15 & 2,0 \\
\hline 19 a 25 & 174 & 23,6 & 116 & 15,8 & 99 & 13,5 & 93 & 12,6 & 88 & 12,0 \\
\hline 26 a 35 & 43 & 5,8 & 22 & 3,0 & 19 & 2,6 & 30 & 4,1 & 18 & 2,4 \\
\hline$\geq 36$ & 6 & 0,8 & 2 & 0,3 & 3 & 0,4 & 4 & 0,5 & 4 & 0,5 \\
\hline$p^{*}$ & \multicolumn{2}{|c|}{0,122} & \multicolumn{2}{|c|}{0,559} & \multicolumn{2}{|c|}{0,892} & \multicolumn{2}{|c|}{$\leq 0,001$} & \multicolumn{2}{|c|}{0,601} \\
\hline \multicolumn{11}{|l|}{ Curso } \\
\hline Educação Física & 3 & 0,4 & 0 & 0,0 & 4 & 0,5 & 9 & 1,2 & 0 & 0,0 \\
\hline Enfermagem & 96 & 13,0 & 59 & 8,0 & 64 & 8,7 & 41 & 5,6 & 53 & 7,2 \\
\hline Fisioterapia & 64 & 8,7 & 32 & 4,3 & 25 & 3,4 & 29 & 3,9 & 16 & 2,2 \\
\hline Nutrição & 83 & 11,7 & 61 & 8,3 & 40 & 5,4 & 52 & 7,1 & 56 & 7,6 \\
\hline$p^{\#}$ & \multicolumn{2}{|c|}{$\leq 0,001$} & \multicolumn{2}{|c|}{$\leq 0,001$} & \multicolumn{2}{|c|}{$\leq 0,001$} & \multicolumn{2}{|c|}{0,603} & \multicolumn{2}{|c|}{$\leq 0,001$} \\
\hline \multicolumn{11}{|l|}{ Semestre letivo } \\
\hline Iniciais & 37 & 5,0 & 20 & 2,7 & 14 & 1,9 & 16 & 2,2 & 16 & 2,2 \\
\hline Intermediários & 152 & 20,7 & 111 & 15,1 & 91 & 12,4 & 86 & 11,7 & 84 & 11,4 \\
\hline Finais & 57 & 7,7 & 21 & 2,9 & 28 & 3,8 & 29 & 3,9 & 25 & 3,4 \\
\hline$p^{*}$ & \multicolumn{2}{|c|}{$\leq 0,001$} & \multicolumn{2}{|c|}{$\leq 0,001$} & \multicolumn{2}{|c|}{$\leq 0,001$} & \multicolumn{2}{|c|}{$\leq 0,001$} & \multicolumn{2}{|c|}{$\leq 0,001$} \\
\hline
\end{tabular}

* Teste do Qui-quadrado; **Jornada de estudos extensa; ${ }^{* * *}$ Jornada de trabalho extensa; \# Teste do Qui-quadrado com correção de yates.

\section{Discussão}

O presente estudo verificou que as barreiras para a prática de AF mais prevalentes em universitários de uma instituição de ensino superior localizada na cidade de Fortaleza, nordeste do Brasil, foram jornada de estudos extensa, falta de energia, falta de companhia, jornada de trabalho extensa e falta de interesse. Os resultados indicaram que estas barreiras estiveram associadas ao semestre letivo, sendo a jornada de estudos extensa a única barreira associada ao sexo e, apenas a jornada de trabalho extensa não esteve associada ao curso. Resultados semelhantes foram encontrados em estudos internacionais ${ }^{15,17}$ e nacionais ${ }^{18,22}$ com universitários.

A jornada de estudos extensa foi apontada como a principal barreira percebida para à prática de $\mathrm{AF}$ pelos universitários do presente estudo, este resultado difere do encontrado em um estudo realizado com universitários sul-africanos que identificou a falta de instalações adequadas como a principal barreira percebida para a prática de $\mathrm{AF}^{9}$. Já no Reino Unido, a preguiça foi tida como a principal barreira ${ }^{15}$. Diferenças nos resultados destes estudos podem estar relacionadas com as características de cada região, principalmente no que se refere ao contexto sociocultural e econômico, o qual cada amostra encontra-se inserida ${ }^{18}$.

Em relação ao presente estudo, tal achado indica uma sobrecarga de conteúdo programático viabilizado pelas universidades, no âmbito do ensino, da pesquisa e da extensão e a inabilidade dos universitários em conciliarem o aumento das obrigações acadêmicas com a manutenção de um estilo de vida fisicamente ativo ${ }^{21}$. Ressaltando também que o excesso de atividade laboral somado à ocupação com 
os estudos, pode colaborar para a falta de tempo para a participação em programas de $\mathrm{AF}$ regulares ${ }^{22}$. Isto justificaria o fato da jornada de trabalho extensa também ser apontada como uma das principais barreiras percebidas no presente estudo. Acrescenta-se ainda que, a maioria dos universitários, para custear sua formação, necessita trabalhar, fazendo com que as pendências acadêmicas e profissionais ocupem boa parte de seu tempo livre ${ }^{13}$. Esses achados ressaltam a necessidade de uma melhor administração do tempo por parte dos universitários para que a AF seja incorporada como uma atividade do cotidiano ${ }^{12}$.

A falta de energia e falta de companhia também figuraram entre as principais barreiras percebidas para a prática de $\mathrm{AF}$ entre os universitários, corroborando com outros estudos ${ }^{6,23}$. A falta de energia pode estar relacionada não somente ao tipo de atividade realizada no trabalho e/ou dia a dia, mas também ao tempo envolvido e às posições adotadas, como estar sentado por longo período de tempo. Assim, pode-se justificar que a jornada de estudos e de trabalho extensas, barreiras também bastante prevalentes no presente estudo, reflitam na percepção da falta de energia como uma barreira percebida para a prática, podendo gerar uma sensação de cansaço físico e dores físicas levando os universitários a procurar o descanso em seu tempo livre ${ }^{23}$.

Em relação a falta de companhia uma revisão sistemática concluiu que os colegas e amigos desempenham um papel importante nos níveis de AF dos jovens, tendo em vista que todos os estudos incluídos encontraram associação positiva entre essas variáveis, por meio principalmente do suporte social ou da presença dos colegas e amigos durante a $\mathrm{AF}^{24}$. A mesma relação também já foi identificada em adultos ${ }^{25}$. Assim, a companhia de outras pessoas parece ser essencial para a prática de $\mathrm{AF}$, independentemente da faixa etária.

Quanto ao sexo houve diferença significativa em relação à jornada de estudos extensa $(27,9 \%$ e $5,6 \%$, mulheres e homens respectivamente, $p \leq 0,004)$. Este resultado apesar de possuir um aspecto negativo para a manutenção de um estilo de vida fisicamente ativo, sugere a atual conjuntura social, na qual a mulher está cada vez mais participativa no mercado de trabalho e nas salas das universidades ${ }^{26}$.

Em relação à faixa etária, verificou-se um elevado número de indivíduos entre 19 e 25 anos relatando a jornada de trabalho extensa como fator limitante para a prática de AF. Possivelmente, a maior percepção de obrigações laborais nesta faixa etária, se deve ao fato de que é nessa fase da vida onde o jovem encontra-se se inserindo no mercado de trabalho, sentindo dificuldades de adaptação a uma nova rotina ${ }^{27}$.

No presente estudo registrou-se uma maior prevalência de mulheres, estudantes do curso de nutrição e enfermagem e estudantes de semestres letivos intermediários que não praticavam AF. O que justifica o fato de serem estes os grupos que mais apresentaram barreiras para a prática de $\mathrm{AF}$. Este resultado reforça a associação entre a percepção de barreiras e a participação em $\mathrm{AF}^{20}$. Nesse contexto, deve-se considerar o aspecto sociocultural sobre os papéis que os diferentes sexos exercem na sociedade, o que muitas vezes acarreta em maior estímulo para a prática de atividades físicas nos meninos, desde a infância ${ }^{9}$. Em relação ao curso este resultado pode estar relacionado à matriz curricular e aos tipos de estágio em cada curso, onde os universitários comprometem-se progressivamente com atividades acadêmicas, extracurricular e extraclasse, fatores estes que podem estar relacionados negativamente com a prática de $\mathrm{AF}^{28}$.

Os universitários do curso de Educação Física foram os únicos que apresentarem um número menor de relatos com relação às principais barreiras percebidas, reforçando achados anteriores ${ }^{13}$. Hipoteticamente os universitários do curso de 
educação física possuem maior probabilidade de se envolver em programas de AF, seja durante o período de estágio curricular ou mesmo atuando profissionalmente antes da conclusão do curso, característica comum nessa profissão. No entanto, ainda é necessário que se investigue se essa maior tendência a prática de AF observada nesse grupo é um reflexo do perfil do curso ou ao conhecimento gerado pelas disciplinas acadêmicas que compõem a grade curricular, ou mesmo pelo perfil individual de cada estudante ingressante no curso.

Os resultados indicaram que as principais barreiras percebidas para a prática de AF estiveram associadas ao semestre letivo, sendo a proporção mais elevada para semestres intermediários. Esses achados divergem do estudo realizado com 5.461 universitários de uma instituição pública do estado da Bahia, em que os universitários dos últimos semestres da graduação relataram um maior número de barreiras percebidas para a prática de $\mathrm{AF}^{16}$. Uma possível explicação deve-se ao fato de que na instituição onde foi realizado o presente estudo, grande parte dos universitários matriculados em semestres finais estarem cursando um menor número de disciplinas e/ou disciplinas na modalidade estudo dirigido, culminando em um menor tempo gasto com atividades acadêmicas. Além, é claro, de um conhecimento mais abrangente e bem estruturado sobre a importância da prática de AF regular para a saúde, o que hipoteticamente deve ter colaborado para a superação de possíveis barreiras ${ }^{30}$.

É importante destacar que mesmo considerando os riscos decorrentes da inatividade física existem poucas investigações relacionadas a barreiras percebidas para a prática de $\mathrm{AF}$ em universitários no Brasil. Um aspecto a ser destacado no presente estudo foi o rigor metodológico do processo de seleção amostral e à padronização dos métodos de coleta de dados, incluindo o rigoroso treinamento dos entrevistadores e o controle de qualidade durante todo o período do trabalho de campo.

Algumas limitações precisam ser consideradas. O fato de não haver questionários específicos para avaliar barreiras para a prática de AF em universitários. Dessa forma optou-se por utilizar um questionário elaborado por Martins e Petroski ${ }^{14}$, acrescentado o item "jornada de estudos extensa", para melhor validade junto aos discentes.

O presente estudo identificou que a jornada de estudos extensa foi a barreira percebida mais prevalente para a prática de $\mathrm{AF}$ entre os universitários e a única barreira associada ao sexo. Registrou-se ainda que o semestre letivo esteve associado as principais barreiras percebidas e que apenas a jornada de trabalho extensa não esteve associada ao curso. Verificou-se ainda que as principais barreiras percebidas eram de cunho social, comportamental e físico.

Mediante os resultados encontrados, sugere-se o desenvolvimento de práticas de $\mathrm{AF}$ e esportivas gratuitas dentro da própria universidade, ofertadas em horários que facilitam a participação dos universitários, bem como o desenvolvimento de ações que objetivem estimular a prática regular de AF.

\section{Contribuição dos autores}

T. P. do Nascimento (0000-0003-2564-5895) participou da concepção inicial do estudo, redação e coleta de dados. F. R. Alves (0000-0001-9105-2356) foi responsável pela busca da literatura e colaborou na revisão crítica do texto. E. A. de Souza (0000-0003-4964-4934) realizou a análise dos dados e revisão crítica do texto. Todos os autores aprovaram a versão final a ser publicada. 


\section{Referências}

1. Ministério da Educação. Evolução do Número de Vagas segundo a Região e a Unidade da Federação - Brasil. Censo da Educação Superior 1991-2007. [citado em: 29 set. 2011]. Disponível em http://portal.inep.gov.br/web/censo-da-educacao-superior/evolucao1980-a-2007.

2. Oliveira CS, Gordia AP, Quadros TMB, Campos W. Physical activities of brazilian university students: a revision of the literature. Rev. Aten. Saúde. 2014; 12 (42): 71-7.

3. Haase A, Steptoe A, Sallis JF, Wardle J. Leisure-time physical activity in university students from 23 countries: associations with health beliefs, risk awareness, and national economic development. Prev Med. 2004; 39:182-90.

4. Silva DAS, Petroski EL. Factors associated with the degree of participation in physical activities among students of a public university in the south of Brazil. Cienc Saude Colet. 2012;16:4087-94.

5. Silva DAS, Magalhães I, Pereira IMM. Estágios de mudança de comportamento para atividade física e fatores associados em acadêmicos de educação física. Rev Bras Ativ Fis Saude 2010; 5(1): 15-20.

6. Elsangedy HM, Krinski K, Buzzachera CF, Cieslak F, Vitorino DC, Silva SG. Nível de atividade física e suas possíveis barreiras em docentes universitários de Toledo-PR. Lecturas Educación Física y Deportes 2008; 12 (1): 05-10.

7. Ceschini FL, Figueira Junior A. Barreiras e determinantes para a prática de atividade física em adolescentes. Rev Bras Ci Mov 2007; 15(1): 29-36.

8. Martins MO. Estudo dos fatores determinantes da prática de atividades físicas de professores universitários [dissertação de mestrado]. Florianópolis: Universidade Federal de Santa Catarina; 2000.

9. Drambos DD, Lopes LFD, Santos DL. Perceived barriers and physical activity in adolescent students from a Southern Brazilian city. Rev Bras Cineantropom Desempenho Hum. 2011, 13(6):422-28.

10. Santos MS, Fermino RC, Reis RS, Cassou AC, Añez CRR. Barreiras para a prática de atividade física em adolescentes: um estudo por grupos focais. Rev Bras Cineantropom Desempenho Hum. 2010; 12 (3): 137-43.

11. Cordeiro PCC, Claumann GS, Pereira EF, Guimarães ACA, Pelegrini A. Estágios de mudança de comportamento relacionados à atividade física em acadêmicos de Educação Física. Rev Bras Ativ Fis Saúde. 2014; 19(4):484-486.

12. Rigoni PAG, Nascimento Junior JRA, Costa GNFS, Vieira LF. Estágios de mudança de comportamento e percepção de barreiras para a prática de atividade física em universitários do curso de Educação Física. Rev Bras Ativ Fís Saúde. 2012; 17(2): 87-92.

13. Mielke GI, Ramis TR, Habeyche EC, Oliz MM, Tessmer MGS, Azevedo MR, et al. Atividade física e fatores associados em universitários do primeiro ano da Universidade Federal de Pelotas. Rev Bras Ativ Fís e Saúde. 2010; 15 (1): 57-4.

14. Martins MO, Petroski EL. Mensuração da percepção de barreiras para a prática de atividades físicas: uma proposta de instrumento. Rev Bras Cineantropom Desempenho Hum. 2000; 2 (1): 58-5.

15. Arzu D, Tuzun EH, Eker L. Perceived barriers to physical activity in university students. J. Sports Sci Med. 2006; 5 (4): 615-4.

16. Awadalla NJ, Aboelyazed AE, Hassanein MA, Khalil SN, Aftab R, Gaballa II, et al. Assessment of physical inactivity and perceived barriers to physical activity among health college students, south-western Saudi Arabia. East Mediterr Health J. 2014; 20 (10): 22-6.

17. Martínez-Lemos RI, Puig RA, García-García O. Perceived Barriers to Physical Activity and Related Factors in Spanish University Students. Am J Prev Med. 2014; 4 (2): 164-74.

18. Lovell GP, Elansari W, Parker JK. Perceived exercise benefits and barriers of nonexercising female university students in the United Kingdom. Int J Environ Res Public Health. 2010; 7(3):784-98.

19. Sousa TFD, Fonseca AS, Barbosa AR. Perceived barriers by university students in relation the leisure-time physical activity. Rev Bras Cineantropom Desempenho Hum. 2013; 15 (2): $164-73$.

20. Souza ECD, Bonfante ILP, Junior FBM, Lopes WA. Níveis de atividade física e estágios de mudança de comportamento de universitários da área de saúde. Rev Bras Ativ Fis Saúde. 2015; 20(6):608-610. 
21. Oliveira CDS, Gordia AP, Quadros TMBD, Campos WD. Atividade física de universitários brasileiros: uma revisão da literatura. Rev Aten. Saúde. 2014; 12 (42): 71-7.

22. Sousa TF, Santos SFDS, José HPM. Barreiras percebidas à prática de atividade física no Nordeste do Brasil. Pensar a Prática. 2010; 13 (1): 12-5.

23. Pinto AA, Claumann GS, Cordeiro PC, Felden EPRG, Pelegrini A. Perceived barriers to physical activity in Physical Education students. Rev Bras Ativ Fís Saúde. 2017;22(1):66-75.

24. Ball K, Bauman A, Leslie E, Owen N. Perceived environmental aesthetics and convenience and company are associated with walking for exercise among Australian adults. Prev Med. 2001; 33(5): 434-40.

25. Ball K, Bauman A, Leslie E, Owen N. Perceived environmental aesthetics and convenience and company are associated with walking for exercise among Australian adults. Prev Med. 2001; 33(5): 434-40.

26. Magalhães B, Silva G. A mulher no trabalho, na família e na universidade. Rev Eletr Arm Crít. 2010; 2 (2):119-3.

27. Hallal PC, Victora CG, Azevedo MR, Wells JCK. Adolescent physical activity and health. Sports Med. 2006; 36 (12):1019-30.

28. Pires CGS, Mussi FC, Cerqueira BB, Pitanga FJG, Silva DO. Prática de atividade física entre estudantes de graduação em enfermagem. Acta Paulista de Enfer. 2013; 26 (5): 436-3.

29. Ramires VV, Becker LA, Sadovsky ADI, Zago AM, Bielemann RM, Gerra PH. Evolução da pesquisa epidemiológica em atividade física e comportamento sedentário no Brasil: atualização de uma revisão sistemática. Rev Bras Ativ Fís Saúde. 2014; 19 (5): 529-47.

Endereço para

Correspondência

Felipe Rocha Alves

proffelipe91@hotmail.com
Programa de Pós-Graduação em

Ciências Médicas. Rua Costa Mendes,

$1608,4^{\circ}$. Andar, Rodolfo Teófilo, CEP

60430-140. Fortaleza, Ceará, Brasil
RECEBIDO 19/08/2016

REVISADO 12/11/2016

APROVADO $14 / 12 / 2016$ 